\title{
Epigallocatechin-3-gallate regulates cell growth, cell cycle and phosphorylated nuclear factor-KB in human dermal fibroblasts
}

\author{
Dong-Wook HAN ${ }^{1, \#}$, Mi Hee LEE ${ }^{2,3, \#}$, Hak Hee KIM², ${ }^{2}$, Suong-Hyu HYON ${ }^{4}$, Jong-Chul PARK², 3, * \\ ${ }^{1}$ Department of Nanomedical Engineering, College of Nanoscience \& Nanotechnology, Pusan National University, Busan 609-735, \\ Korea; ${ }^{2}$ Cellbiocontrol Laboratory, Department of Medical Engineering, ${ }^{3}$ Brain Korea 21 Project for Medical Science, Yonsei University \\ College of Medicine, Seoul 120-752, Korea; ${ }^{4}$ Department of Medical Simulation Engineering, Research Center for Nano Medical Engi- \\ neering, Institute for Frontier Medical Sciences, Kyoto University, Kyoto 606-8507, Japan
}

Aim: To investigate the effects of (-)epigallocatechin-3-gallate (EGCG), the main polyphenol in green tea, on cell growth, cell cycle and phosphorylated nuclear factor-kB ( $\mathrm{pNF-kB}$ ) expression in neonatal human dermal fibroblasts (nHDFs).

Methods: The proliferation and cell-cycle of nHDFs were determined using WST-8 cell growth assay and flow cytometry, respectively. The apoptosis was examined using DNA ladder and Annexin V-FITC assays. The expression levels of pNF-kB and cell cycle-related genes and proteins in nHDFs were measured using cDNA microarray analyses and Western blot. The cellular uptake of EGCG was examined using fluorescence (FITC)-labeled EGCG (FITC-EGCG) in combination with confocal microscopy.

Results: The effect of EGCG on the growth of nHDFs depended on the concentration tested. At a low concentration (200 $\mu$ mol/L), EGCG resulted in a slight decrease in the proportion of cells in the $S$ and $G_{2} / M$ phases of cell cycle with a concomitant increase in the proportion of cells in $\mathrm{G}_{0} / \mathrm{G}_{1}$ phase. At the higher doses (400 and $800 \mu \mathrm{mol} / \mathrm{L}$ ), apoptosis was induced. The regulation of EGCG on the expression of pNF-kB was also concentration-dependent, whereas it did not affect the unphosphorylated NF-kB expression. cDNA microarray analysis showed that cell cycle-related genes were down-regulated by EGCG (200 $\mu \mathrm{mol} / \mathrm{L})$. The expression of cyclins A/B and cyclin-dependent kinase 1 was reversibly regulated by EGCG $(200 \mu \mathrm{mol} / \mathrm{L})$. FITC-EGCG was found to be internalized into the cytoplasm and translocated into the nucleus of nHDFs.

Conclusion: EGCG, through uptake into cytoplasm, reversibly regulated the cell growth and expression of cell cycle-related proteins and genes in normal fibroblasts.

Keywords: (-)epigallocatechin-3-gallate (EGCG); polyphenol; green tea; cell cycle; fibroblasts; nuclear factor-kB (NF-kB)

Acta Pharmacologica Sinica (2011) 32: 637-646; doi: 10.1038/aps.2011.17; published online 25 Apr 2011

\section{Introduction}

Food bioactives with strong antioxidant properties have been shown to be contained in green tea, grapes, and turmeric and have shown cancer chemopreventive and chemotherapeutic effects in many animal tumor bioassays, cell culture systems, and epidemiological studies ${ }^{[1-3]}$. These biological activities of green tea polyphenols are believed to be mostly mediated by (-)epigallocatechin-3-gallate (EGCG), the predominant catechin present therein ${ }^{[4]}$. Some previous reports have shown that treatment with EGCG or its derivatives results in $\mathrm{G}_{0} / \mathrm{G}_{1}$ phase cell cycle arrest and apoptosis of several types of cancer

\footnotetext{
\# These authors contributed equally to this paper.

* To whom correspondence should be addressed.

E-mail parkjc@yuhs.ac

Received 2010-09-13 Accepted 2011-02-14
}

cells, but not of normal cells ${ }^{[5-7]}$. However, the mechanism of this differential response to EGCG in cancer cells vs normal cells has not been fully elucidated. Many previous studies have reported the incorporation of EGCG into the cytosol and/or even the nucleus of cancer cells. The EGCG tissue distribution and metabolism in animals has also been assessed using radioisotope-labeled EGCG, eg, $\left[{ }^{3} \mathrm{H}\right] \mathrm{EGCG}{ }^{[8-11]}$. This cellular incorporation of EGCG is considered involved in the mechanism of the anti-cancer activity of EGCG because catechins exert proapoptotic and antiproliferative activities on cancer cells.

Dietary agents that can suppress the cancer cells via apoptosis (a programmed cell death) but do not affect normal cells may have a therapeutic advantage for the elimination of cancer cells. At present, only a few agents are known to possess the potential for selective/preferential elimination of cancer 
cells without affecting the normal cells ${ }^{[6,7,12,13]}$. EGCG has been shown to have suppressive effects on various cancer cells by inducing cell cycle arrest and inhibiting proliferation ${ }^{[14,15]}$, whereas less attention has been paid to its cytoprotective effects on normal cells. Our previous reports have demonstrated that EGCG induces cellular hibernation with high cell survival rates through inhibiting apoptosis by scavenging free radicals harmful to the cells and protecting the fragile cell membrane by simply adhering to and reinforcing it ${ }^{[16-18]}$.

In the present study, we investigated (i) the regulatory effects of low- and high-dose treatments of EGCG on the cellular responses and expression of phosphorylated nuclear factor-kB/p65 (pNF-kB/p65) in neonatal human dermal fibroblasts (nHDFs), (ii) the reversible effects of EGCG on the expression of cell cycle-related genes and proteins, and (iii) the involvement of the cellular uptake pattern of EGCG as a mechanism for these dose-differential responses to EGCG in nHDFs. Our data imply that the proliferation, cell cycle progression, apoptosis and $\mathrm{pNF}-\mathrm{kB} / \mathrm{p} 65$ expression of $\mathrm{nHDFs}$ are dose-differentially regulated in response to EGCG (200$800 \mu \mathrm{mol} / \mathrm{L})$. Cell cycle-related genes and proteins, such as cyclin A (CCNA), CCNB, cyclin-dependent kinase 1 (CDK1) and CDK inhibitor A1 (CDKN1A or p21), were reversibly regulated when the cells were subjected to EGCG exposure and removal. EGCG conjugated with fluorescein isothiocyanate (FITC) was found to be incorporated into the cytoplasm of nHDFs with further nuclear translocation. The dose-differential regulatory activity of EGCG may be exploited to craft strategies for the cytoprotection of normal cells and cancer chemoprevention by EGCG.

\section{Materials and methods}

\section{Cell cultures}

Neonatal human dermal fibroblasts (nHDFs) were kindly provided by Dr Dong Kyun RAH (Department of Plastic and Reconstructive Surgery, Yonsei University College of Medicine, Seoul, Korea). The cells were routinely maintained in Dulbecco's modified Eagle's medium (D6429, Sigma-Aldrich Co, St Louis, MO, USA) supplemented with $10 \%$ fetal bovine serum (Sigma-Aldrich Co) and a 1\% antibiotic antimycotic solution (including 10000 units penicillin, $10 \mathrm{mg}$ streptomycin and $25 \mu \mathrm{g}$ amphotericin B per mL, Sigma-Aldrich Co) at 37 ${ }^{\circ} \mathrm{C}$ in a humidified atmosphere of $5 \% \mathrm{CO}_{2}$ in air as previously described $^{[19]}$. Studies were performed with nHDFs within 10 passages.

\section{EGCG treatment}

EGCG (Teavigo ${ }^{\mathrm{TM}}$ ), a major polyphenolic constituent of green tea, was purchased from DSM Nutritional Products Ltd (Basel, Switzerland), and its purity exceeded $98 \%$. To examine the dose-based differential effects of EGCG on growth, cell cycle progression, apoptosis and $\mathrm{pNF}-\mathrm{kB} / \mathrm{p} 65$ expression of $\mathrm{nHDFs}$, the cells were seeded into well plates and incubated in the presence of increasing concentrations (100-800 $\mu \mathrm{mol} / \mathrm{L})$ of EGCG in complete medium for $24 \mathrm{~h}$. Upon determining the effects of EGCG on the expression of cell cycle-related genes or proteins, cultured cells were treated with $200 \mu \mathrm{mol} / \mathrm{L}$ EGCG in complete medium for $24 \mathrm{~h}$. After EGCG treatment, EGCG was removed from the culture medium and the cells were further incubated for 24 to $72 \mathrm{~h}$.

\section{Cell growth assay}

The number of viable cells was quantified indirectly using a highly water soluble tetrazolium salt [WST-8, 2-(2-methoxy4-nitrophenyl)-3-(4-nitrophenyl)-5-(2,4-disulfophenyl)-2Htetrazolium, monosodium salt] (Dojindo Lab, Kumamoto, Japan) that is reduced to a formazan dye by mitochondrial dehydrogenases. Cell growth was found to be directly proportional to the metabolic reaction products obtained in the WST-8 assay. Briefly, WST-8 assays were conducted as follows. nHDFs cultures treated with increasing concentrations of EGCG were incubated with WST- 8 for the last $4 \mathrm{~h}$ of the culture period $(24 \mathrm{~h})$ at $37^{\circ} \mathrm{C}$ in the dark. To avoid a direct reaction between the antioxidant EGCG and the WST- 8 to be reduced, the cell cultures were thoroughly washed with phosphate-buffered saline (PBS, $\mathrm{pH}$ 7.2) and refreshed with a fresh medium containing WST-8. Parallel sets of wells containing freshly cultured, non-treated nHDFs were regarded as the controls. Absorbance was determined at $450 \mathrm{~nm}$ using an ELISA reader (SpectraMax ${ }^{\odot}$ 340, Molecular Device Co, Sunnyvale, CA, USA). At the end of incubation, the cellular morphologies were observed under an Olympus IX70 inverted microscope (Olympus Optical Co, Osaka, Japan).

\section{Cell cycle analysis}

For cell cycle analysis, cultured nHDFs, following treatment with increasing concentrations of EGCG for $24 \mathrm{~h}$, were collected and washed with cold PBS ( $\mathrm{pH} 7.2)$. The cells were resuspended in $95 \%$ cold methanol for $1 \mathrm{~h}$ at $4{ }^{\circ} \mathrm{C}$ and then centrifuged at $120 \times g$ for $5 \mathrm{~min}$. The resultant pellet was washed twice with cold PBS, suspended in PBS and incubated with RNase (20 Units/mL, final concentration, Sigma-Aldrich Co) at $37^{\circ} \mathrm{C}$ for $30 \mathrm{~min}$. Afterward, the cells were chilled on ice for $10 \mathrm{~min}$ and stained with $100 \mu \mathrm{g} / \mathrm{mL}$ propidium iodide (PI, Sigma-Aldrich Co) for $1 \mathrm{~h}$. At least 10000 cells were counted by a flow cytometer (FACSCalibur ${ }^{\mathrm{TM}}$, BD Biosciences, San Jose, CA, USA), and the data obtained were analyzed using ModFit ${ }^{\mathrm{TM}}$ LT for Mac version 3.0 software (Verity Software House, Topsham, ME, USA).

\section{Apoptosis analysis}

After treatment of nHDFs with increasing concentrations of EGCG for $24 \mathrm{~h}$, the cellular DNA was isolated and resolved over a $1.5 \%$ agarose gel to assess DNA ladder formation (fragmentation) using a standard procedure. Briefly, following EGCG treatment, the cells were washed twice with PBS ( $\mathrm{pH}$ 7.2) and incubated with $1.0 \mathrm{~mL}$ of cytoplasm extraction buffer (10 mmol/L Tris, pH 7.5, $150 \mathrm{mmol} / \mathrm{L} \mathrm{NaCl}, 5 \mathrm{mmol} / \mathrm{L} \mathrm{MgCl}_{2}$, and $0.5 \%$ Triton $\mathrm{X}-100)$ on ice for $15 \mathrm{~min}$. The cells were centrifuged $(14000 \times g)$ at $4{ }^{\circ} \mathrm{C}$ and then incubated with $1.0 \mathrm{~mL}$ of DNA lysis buffer (10 mmol/L Tris, pH 7.5, $400 \mathrm{mmol} / \mathrm{L} \mathrm{NaCl}$, $1 \mathrm{mmol} / \mathrm{L}$ EDTA, and 1\% Triton X-100) for $20 \mathrm{~min}$ on ice. The 
lysate was cleared by centrifugation $(14000 \times g)$ at $4{ }^{\circ} \mathrm{C}$, and the supernatant was incubated overnight with RNase $(0.2 \mathrm{mg} / \mathrm{mL})$ at room temperature and then with Proteinase $\mathrm{K}(0.1 \mathrm{mg} / \mathrm{mL}$, Sigma-Aldrich Co) for $2 \mathrm{~h}$ at $37^{\circ} \mathrm{C}$. DNA was extracted using phenol:chloroform (1:1) and precipitated with 95\% ethanol. The DNA precipitate was centrifuged at $14000 \times g\left(4{ }^{\circ} \mathrm{C}\right)$ for 15 $\mathrm{min}$, and the pellet was air-dried and dissolved in $20 \mathrm{~mL}$ of TE buffer (10 mmol/L Tris-HCl, pH 8.0, and $1 \mathrm{mmol} / \mathrm{L}$ EDTA). The total amount of DNA obtained was resolved over a $1.5 \%$ agarose gel containing $0.3 \mathrm{mg} / \mathrm{mL}$ ethidium bromide in Trisborate-EDTA buffer. The bands were visualized under a UV transilluminator followed by Polaroid photography.

Apoptosis of nHDFs treated with EGCG for $24 \mathrm{~h}$ was also determined by the TACS ${ }^{\mathrm{TM}}$ Annexin V-FITC assay (R\&D Systems Inc, McKinley Place, MN, USA). Phosphatidylserines exposed on the membrane surface of apoptotic cells were stained with Annexin V-FITC according to the manufacturer's instructions. The late apoptotic (or necrotic) cells were stained with PI. Parallel sets of wells containing non-treated nHDFs were regarded as the $(+)$ controls. The results were acquired and analyzed with CellQuest ${ }^{\odot}$ software (BD Biosciences). The population of apoptotic cells was characterized by its high mean fluorescence of Annexin V-FITC in a flow cytometric histogram.

\section{Western blotting}

After treatment with increasing concentrations of EGCG for $24 \mathrm{~h}, \mathrm{nHDF}$ were washed twice with cold PBS $(10 \mathrm{mmol} / \mathrm{L}$, pH 7.4), and ice-cold RIPA lysis buffer (Santa Cruz Biotechnology Inc, Santa Cruz, CA, USA) was added to the cells. After $5 \mathrm{~min}$, the cells were scraped, and the lysate was centrifuged at $14000 \times \mathrm{g}$ for $20 \mathrm{~min}$ at $4{ }^{\circ} \mathrm{C}$. Proteins were extracted from the total lysate, and the protein concentration was determined by a $\mathrm{BCA}^{\mathrm{TM}}$ protein assay using the manufacturer's protocol (Pierce, Rockford, IL, USA). For immunoblot analysis, 35-40 $\mu \mathrm{g}$ of protein was run on a 4/20 polyacrylamide-SDS gel (Daiichi Pure Chemicals Co, Ltd, Tokyo, Japan) for $1 \mathrm{~h}$ at $30 \mathrm{~mA}$ and blotted to a PVDF membrane for $50 \mathrm{~min}$ at $35 \mathrm{~mA}$. The membrane was blocked in a blocking buffer (Nacalai Tesque Inc, Kyoto, Japan) for $1 \mathrm{~h}$ at room temperature and incubated with rabbit anti-human NF-kB/p65 and pNF-kB/p65 (Ser 536) polyclonal antibodies (Cell Signaling Technology Inc, Danvers, MA, USA) and, as the reference, mouse anti-human glyceraldehyde-3-phosphate dehydrogenase (GAPDH) monoclonal antibody (International Inc, Temecula, CA, USA). The membrane was treated with either an anti-mouse IgG (Amersham Biosciences, Buckinghamshire, UK) or an anti-rabbit IgG secondary antibody (Santa Cruz Biotechnology Inc), horseradish peroxidase-conjugated. Protein expression was detected by a Chemilumi-one chemiluminescent kit (Nacalai Tesque Inc) and X-ray film (Fujifilm, Tokyo, Japan).

In the cases of immunoblot analysis for cell cycle-related proteins, such as CCNA, CCNB1, CDK1, and CDKN1A, nHDFs were treated with $200 \mu \mathrm{mol} / \mathrm{L}$ EGCG for $24 \mathrm{~h}$; the EGCG was then removed for further incubation for up to $72 \mathrm{~h}$. Afterward, each protein was extracted from the cell lysate and blotted to the membrane. The membrane was blocked and incubated with primary antibodies, including either mouse anti-human CCNA, CCNB1, and CDK1 monoclonal antibodies (BD Biosciences) or rabbit anti-human CDKN1A monoclonal antibody (Cell Signaling Technology Inc), followed by incubation with horseradish peroxidase-conjugated secondary antibodies. Protein expression was detected as described above.

\section{Microarray analysis}

The effect of EGCG on gene expression of nHDFs was investigated using a Platinum Human Cancer 3.0K oligo microarray (GenoCheck, Ansan, Gyunggi-do, Korea). This microarray consisted of 3096 oligonucleotide spots including Operon (Operon, Huntsville, AL, USA), human oligo subsets, housekeeping genes and Arabidopsis DNA as controls. All oligonucleotide probes were designed from the UniGene Database Build Hs 184 and the Human Reference Sequence Database, both developed and maintained at the National Center for Biotechnology Information. The probes were resuspended in spotting solution (GenoCheck) at a final concentration of $50 \mathrm{pmol} / \mathrm{L}$ and spotted onto CMT-GAPS II silane slide glass (Corning, NY, USA) with a PixSys 5500 arrayer (Cartesian Technologies, Irvine, CA, USA) using 6 stealth micro spotting pins. The printed slides were processed according to the CMT-GAPS II slide protocol.

\section{Confocal laser scanning microscopy}

To determine the cellular uptake patterns of EGCG in nHDFs upon treatment with low and high doses of EGCG, the cells were treated for $24 \mathrm{~h}$ with 100 or $400 \mu \mathrm{mol} / \mathrm{L}$ FITC-conjugated EGCG (FITC-EGCG) as described previously ${ }^{[20]}$. After FITCEGCG treatment, the cells were washed thoroughly with PBS, fixed with 3.5\% paraformaldehyde (Sigma-Aldrich Co) in 0.1 $\mathrm{mol} / \mathrm{L}$ phosphate buffer ( $\mathrm{pH} 7$ ) for $5 \mathrm{~min}$ at room temperature and immediately observed under a confocal laser scanning microscope (LSM 510, Carl Zeiss Advanced Imaging Microscopy, Jena, Germany). Cell nuclei were counterstained with 5 $\mu \mathrm{mol} / \mathrm{L}$ PI immediately before 3 to $5 \mathrm{~min}$ of observation.

\section{Statistical analyses}

All variables were tested in three independent cultures for each experiment, and each experiment was repeated twice $(n=6)$. The results are reported as the mean \pm standard deviation (SD) compared with the non-treated controls. A oneway analysis of variance (ANOVA), which was followed by a Tukey HSD test for the multiple comparisons, was used to detect the dose-based differential effects of EGCG on nHDFs. A $P$ value $<0.05$ was considered statistically significant.

\section{Results}

Effects of EGCG on growth, morphology and cell cycle progression of nHDFs

Proliferation was inhibited in $\mathrm{nHDFs}$ in a dose-differential manner in response to EGCG treatment (Figure 1A). The cells partially lost their proliferation feedback control mechanism during EGCG treatment with lower than $200 \mu \mathrm{mol} / \mathrm{L}$ for $24 \mathrm{~h}$, 
but the proliferation control was slowly recovered by the cell to cell contact and returned to a normal level after the removal of EGCG from the medium (data not shown). However, upon treatment with greater than $400 \mu \mathrm{mol} / \mathrm{L} \mathrm{EGCG}$, the proliferation control was not recovered in spite of EGCG removal.

A dose-differential modulation of cell cycle progression of nHDFs by EGCG treatment was also found. As shown in Figure 1B, EGCG treatment triggered an appreciable dosedependent increase in the Sub-G $\mathrm{G}_{1}$ phase (ie, 5.4\%, 56.7\%, and $69.9 \%$ at $200 \mu \mathrm{mol} / \mathrm{L}, 400 \mu \mathrm{mol} / \mathrm{L}$, and $800 \mu \mathrm{mol} / \mathrm{L}$, respectively), which corresponds to apoptotic cells, and a concomitant decrease in $S$ phase cells. Interestingly, $200 \mu \mathrm{mol} / \mathrm{L}$ EGCG resulted in a slight decrease in the population of $S$ and $\mathrm{G}_{2} / \mathrm{M}$ phases of the cell cycle, leading to cell cycle delay at the $\mathrm{G}_{0} / \mathrm{G}_{1}$ phase. However, EGCG treatment at a concentration greater than $400 \mu \mathrm{mol} / \mathrm{L}$ resulted in a significant increase in the $\mathrm{Sub}-\mathrm{G}_{1}$ phase of the cell cycle with a remarkable decrease in the proportion of cells in both the $S$ and the $G_{2} / M$ phases. Accordingly, the cells could not enter the $S$ phase during treatment with higher concentrations of EGCG, which might induce cell cycle arrest as well as apoptosis.

This dose-differential antiproliferative response of EGCG in nHDFs was evident from the morphological observations (Figure $1 \mathrm{C}$ ). The non-treated control and the lower doses (100 and $200 \mu \mathrm{mol} / \mathrm{L}$ ) of EGCG caused only slight alterations, if any, in the cellular morphologies of nHDFs. At the higher doses
(400 and $800 \mu \mathrm{mol} / \mathrm{L}$ ), however, the number of attached cells was markedly decreased, indicating that the EGCG treatments might result in apoptotic detachment of the cells.

\section{Effects of EGCG on apoptosis of nHDFs}

The dose-differential effects of EGCG on apoptosis of nHDFs were evident from a DNA ladder assay, which showed the induction of DNA fragmentation with increasing concentrations of EGCG treatment (Figure 2A). The Annexin V-FITC assay showed that neither apoptosis nor necrosis was seen at doses lower than $200 \mu \mathrm{mol} / \mathrm{L}$ EGCG (Figure 2B). However, treatment of nHDFs with higher concentrations of EGCG resulted in late apoptosis and necrosis as evident by the formation of internucleosomal DNA fragments and high fluorescence of Annexin V-FITC and PI. Overall, this dose-differential pattern of apoptosis induced by EGCG in nHDFs agreed well with that of cell growth regulation, as shown in Figure 1.

\section{Effects of EGCG on pNF-kB/p65 expression in nHDFs}

To evaluate differential effects of EGCG on the expression of constitutive NF-kB/p65 and pNF-kB/p65 in nHDFs, an antibody directed against the RelA/p65 subunit of each protein was employed. EGCG treatment did not result in any significant alternation in the inactive, unphosphorylated form of NF-kB/p65 protein expression (Figure 3). However, a very strong inhibition of $\mathrm{pNF}-\mathrm{kB} / \mathrm{p} 65$ expression was observed at

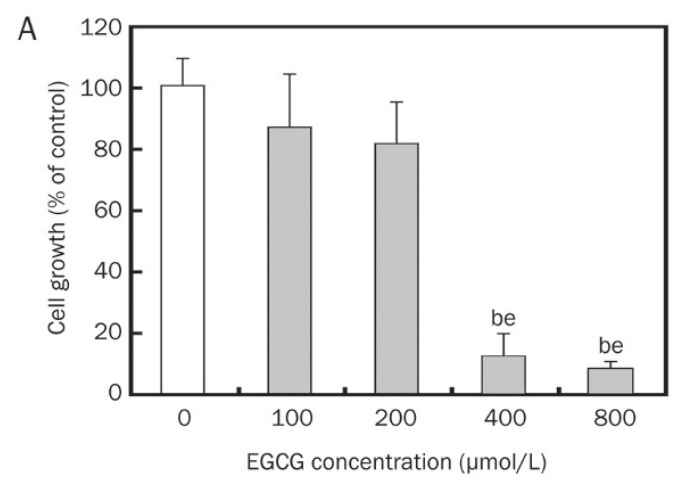

C $\operatorname{EGCG}(\mu \mathrm{mol} / \mathrm{L})$
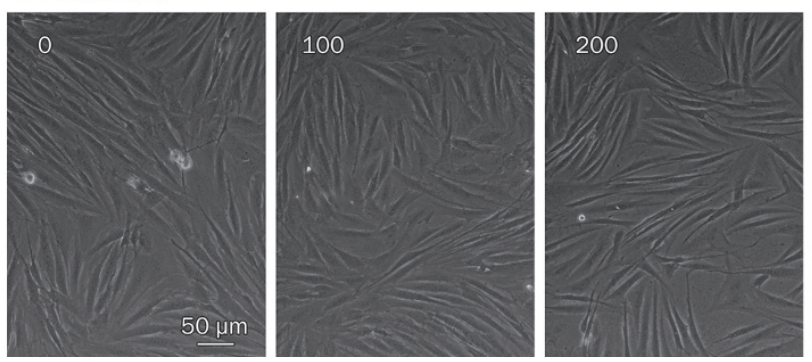

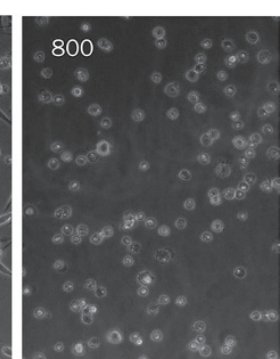

Figure 1. Dose-based differential effects of EGCG on proliferation, morphology, and cell cycle progression of nHDFs. Cells were incubated with increasing concentrations (100-800 $\mu \mathrm{mol} / \mathrm{L})$ of EGCG for $24 \mathrm{~h}$. The proliferation (A), cell cycle progression (B), and morphology (C) of EGCG-treated nHDFs were measured by WST-8 assay, flow cytometry and optical microscopy, respectively. The results are reported as a mean \pm SD and analyzed by a Tukey HSD test. ${ }^{\mathrm{b}} P<0.05$ vs non-treated control, ${ }^{e} P<0.05$ vs 100 or $200 \mu \mathrm{mol} / \mathrm{L}$ EGCG group, respectively (A and B). The data shown in this figure are representative of 6 independent experiments, showing similar results. 
A

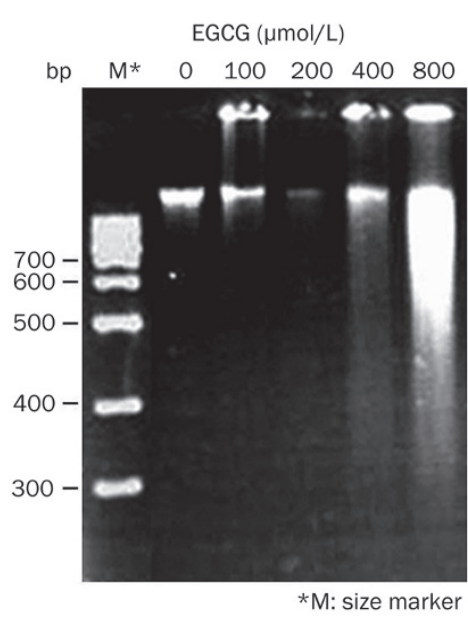

B

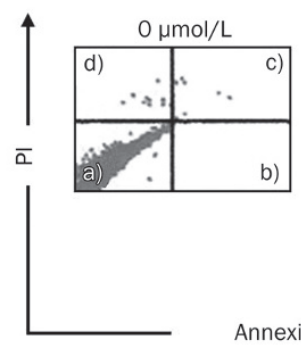

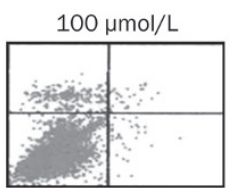

$400 \mu \mathrm{mol} / \mathrm{L}$

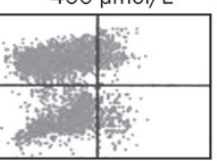

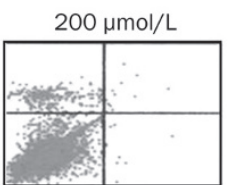

$800 \mu \mathrm{mol} / \mathrm{L}$

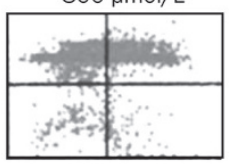

Annexin V-FITC

\begin{tabular}{ccccc}
\hline $\begin{array}{c}\text { EGCG } \\
(\mu \mathrm{mol} / \mathrm{L})\end{array}$ & Survival $^{\text {a) }}$ & \multicolumn{2}{c}{ Apoptosis } & Necrosis $^{\mathrm{d})}$ \\
\hline 0 & $98.0 \pm 4.4$ & $0.1 \pm 0.0$ & $0.2 \pm 0.1$ & $1.7 \pm 1.1$ \\
100 & $96.7 \pm 8.1$ & $0.4 \pm 0.6$ & $0.3 \pm 1.1$ & $2.6 \pm 4.1$ \\
200 & $97.0 \pm 7.0$ & $0.1 \pm 1.4$ & $0.2 \pm 0.7$ & $2.7 \pm 1.9$ \\
400 & $32.0 \pm 5.1^{\text {be }}$ & $3.7 \pm 2.5^{\text {be }}$ & $5.3 \pm 9.1^{\text {be }}$ & $59.0 \pm 13.4^{\text {be }}$ \\
800 & $2.2 \pm 1.7^{\text {be }}$ & $1.5 \pm 3.3^{\text {be }}$ & $35.9 \pm 11.6^{\text {be }}$ & $60.4 \pm 8.8^{\text {be }}$ \\
\hline
\end{tabular}

Figure 2. Dose-based differential apoptotic responses of nHDFs to EGCG. Cells were incubated with increasing concentrations (100-800 $\mu$ mol/L) of EGCG for $24 \mathrm{~h}$. DNA fragmentation (A) and apoptosis (B) in EGCG-treated nHDFs were measured by DNA ladder and Annexin V-FITC assays, respectively. The results are reported as a mean \pm SD and analyzed by a Tukey HSD test. ${ }^{b} P<0.05$ vs non-treated control, ${ }^{e} P<0.05$ vs 100 or $200 \mu$ mol/L EGCG group, respectively (lower table in B). The data shown in this figure are representative of 6 independent experiments, showing similar results.

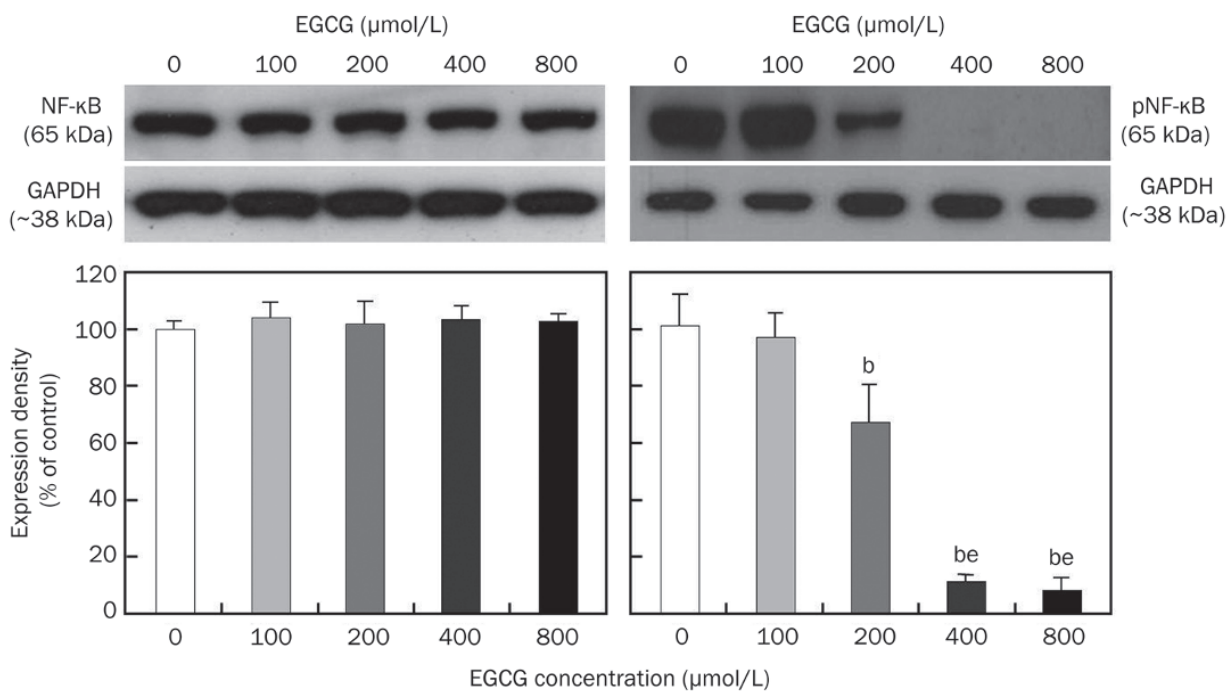

Figure 3. Dose-based differential modulation of pNF-kB expression in nHDFs by EGCG. Cells were incubated with increasing concentrations (100-800 $\mu \mathrm{mol} / \mathrm{L})$ of EGCG for $24 \mathrm{~h}$. Expression levels of NF-KB/p65 and pNF-kB/p65 proteins in EGCG-treated nHDFs were detected by immunoblot analysis as described in Materials and methods. Results from representative experiments were normalized to GAPDH expression by densitometry. The quantitative results are shown in the lower panel. The results are reported as a mean \pm SD and analyzed by a Tukey HSD test. ${ }^{\mathrm{b}} \mathrm{P}<0.05$ vs non-treated control, ${ }^{e} P<0.05$ vs 100 or $200 \mu \mathrm{mol} / \mathrm{L}$ EGCG group, respectively. higher concentrations (400 and $800 \mu \mathrm{mol} / \mathrm{L}$ ) of EGCG, while at $200 \mu \mathrm{mol} / \mathrm{L}$, slight inhibition of pNF-kB/p65 expression occurred. This EGCG-mediated pattern of regulation of the pNF-kB/p65 in the cells was somewhat similar to the pattern of regulation of proliferation. These results suggest that $\mathrm{pNF}-\mathrm{kB} / \mathrm{p} 65$ activation and translocation may be dose-differentially regulated by EGCG.

Effect of EGCG on the expression of cell cycle-related genes and proteins in nHDFs

A cDNA microarray analysis was employed to evaluate the effect of EGCG on the expression level of cell cycle-related genes. The microarray contained oligonucleotide probes representing 3096 human genes and included 50 cell cyclerelated genes. Although a total of 3096 genes were screened in EGCG-treated nHDFs, the expression of 1520 genes was statistically reliable with respect to the control. The expression of only 23 genes was more than 2-fold up-regulated and that of only 61 genes was 2-fold down-regulated in response to 200 $\mu \mathrm{mol} / \mathrm{L}$ EGCG compared with non-treated cells. Among the cell cycle-related genes, some genes were significantly downregulated in response to EGCG treatment (Table 1). These 
Table 1. List of significantly down-regulated/up-regulated cell cycle-related genes in nHDFs after EGCG treatment.

\begin{tabular}{lll}
\hline \multicolumn{1}{c}{ Gene (Symbol) } & Role \\
\hline Cyclin A2 (CCNA2) & Essential for S phase \& $\mathrm{G}_{2} / \mathrm{M}$ phase transition & Fold \\
Cyclin B1 \& B2 (CCNB1 \& CCNB2) & Essential for mitosis, associates with cell division cycle 2 (CDC2) & 0.2 \\
Cyclin-dependent kinase 1 (CDK1 or CDC2) & Complexes with cyclin A \& B, essential for $\mathrm{G}_{2} / \mathrm{M}_{\text {phase transition }}$ & 0.2 \\
Protein kinase, membrane associated tyrosine/threonine 1 (PKMYT1) & Inactivates CDC2 and negatively regulates cell cycle $\mathrm{G}_{2} / \mathrm{M} \mathrm{transition}^{0}$ & 0.3 \\
CDK inhibitor 1A (CDKN1A or p21) & Inhibits cyclin-CDK2 or -CDK4 complexes, regulates $\mathrm{G}_{1}$ phase & 3.3 \\
\hline
\end{tabular}

genes included cyclin (CCN) A2, CCNB1/B2 and their regulatory kinase, cyclin-dependent kinase 1 (CDK1), which function at $S$ phase and the $G_{2} / M$ phase transition. Protein kinase, membrane associated tyrosine/threonine 1 (PKMYT1), which inactivates the cell division cycle 2 (CDC2 or CKD1) protein and negatively regulates the cell cycle $\mathrm{G}_{2} / \mathrm{M}$ transition, was also significantly down-regulated. In contrast, CDK inhibitor A1 (CDKN1A or p21), a $\mathrm{G}_{1}$ phase regulator, was significantly up-regulated upon EGCG treatment. Other cell cycle-related genes, such as CCNC, CCND3, CCNF, CDC20, and CKS2 (CDC28 protein kinase regulatory subunit 2), were slightly down-regulated (Table 2). Interestingly, CDK2 and CDK6, which complex with CCNE and CCND, respectively, and are essential for the $G_{1} / S$ phase transition and early $G_{1}$ phase, were not affected by EGCG treatment. The expression levels of the other cell cycle-related genes are listed in Supplementary Table S1. The detailed expression levels of other genes significantly changed by EGCG treatment relative to the controls are listed in Supplementary Tables S2 and S3.

Figure 4 demonstrates the regulatory effects of EGCG on the expression level of cell cycle-related proteins in nHDFs. The protein expression levels of CCNA, CCNB1, and CDK1 were significantly decreased by $200 \mu \mathrm{mol} / \mathrm{L}$ EGCG treatment for $24 \mathrm{~h}$, but they were noticeably restored after $72 \mathrm{~h}$ of EGCG removal. In contrast, the expression level of CDKN1A was slightly suppressed after EGCG treatment, but remained at low levels $72 \mathrm{~h}$ after the removal of EGCG. These data suggest that the expression of cell cycle-related genes and proteins can be reversibly regulated by EGCG exposure and removal. These results agreed well with those of the cell cycle profile, which showed that the cell cycle of cells treated with EGCG for $24 \mathrm{~h}$ was delayed at $\mathrm{G}_{0} / \mathrm{G}_{1}$ phase and restored after EGCG removal.

\section{Cellular uptake of FITC-EGCG in nHDFs}

To determine and compare the incorporation of FITC-EGCG into the cytoplasm of nHDFs and its further nuclear translocation between low- and high-dose treatments, confocal microscopy was performed in the cells treated with 100 and 400 $\mu \mathrm{mol} / \mathrm{L}$ FITC-EGCG. The cellular uptake of FITC-EGCG was not observed until approximately $4 \mathrm{~h}$ of culture and was most dramatic between 4 and $6 \mathrm{~h}$ (data not shown). As the incubation time progressed, FITC-EGCG became concentrated in the cytoplasm and then localized to different sites within the cells. Although the cellular response to FITC-EGCG and its binding pattern to receptors would be different from those of EGCG, the low dose $(100 \mu \mathrm{mol} / \mathrm{L})$ of FITC-EGCG was clearly observed to be adsorbed onto the membrane and internalized into the cytoplasm of nHDFs, particularly with intense bright green fluorescence after $24 \mathrm{~h}$ (Figure 5A). At higher magnification, FITC-EGCG was clearly found to be widely distributed in the cytosol of the cells and partially translocated into the nucleus, bound to endosome-like structures. However, the incorporation of FITC-EGCG into the cells was appreciably decreased by pretreatment with free EGCG (data not shown), suggesting that a negligible amount of conjugate accumulates

Table 2. List of minimally regulated or unaffected cell cycle-related genes in nHDFs after EGCG treatment

\begin{tabular}{|c|c|c|}
\hline Gene (Symbol) & Role & Fold \\
\hline Cyclin C (CCNC) & Important regulator of $\mathrm{G}_{1}$ phase & 0.8 \\
\hline Cyclin D3 (CCND3) & Essential for $\mathrm{G}_{1}$ phase, with $\mathrm{CDK} 4$ and $\mathrm{CDK} 6$ & 0.7 \\
\hline Cyclin F (CCNF) & Important regulator of cell cycle transitions & 0.6 \\
\hline Cyclin T1 (CCNT1) & Functions as regulators of CDKs & 0.9 \\
\hline Cyclin-dependent kinase 9 (CDK9) & Complexes with CCNT and CCNK & 0.9 \\
\hline Cyclin-dependent kinase 10 (CDK10) & Regulator of $\mathrm{G}_{2} / \mathrm{M}$ phase & 0.9 \\
\hline Cell division cycle 20 (CDC20) & Functions at multiple points in the cell cycle & 0.7 \\
\hline Cell division cycle 23 (CDC23) & Essential for cell cycle progression through the $\mathrm{G}_{2} / \mathrm{M}$ transition & 0.9 \\
\hline CDC28 protein kinase regulatory subunit 2 (CKS2) & Binds to CDKs, essential for their biological functions & 0.6 \\
\hline
\end{tabular}


A

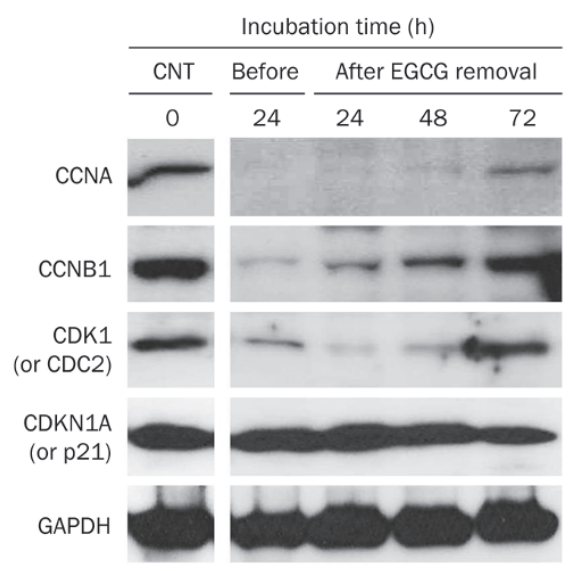

B
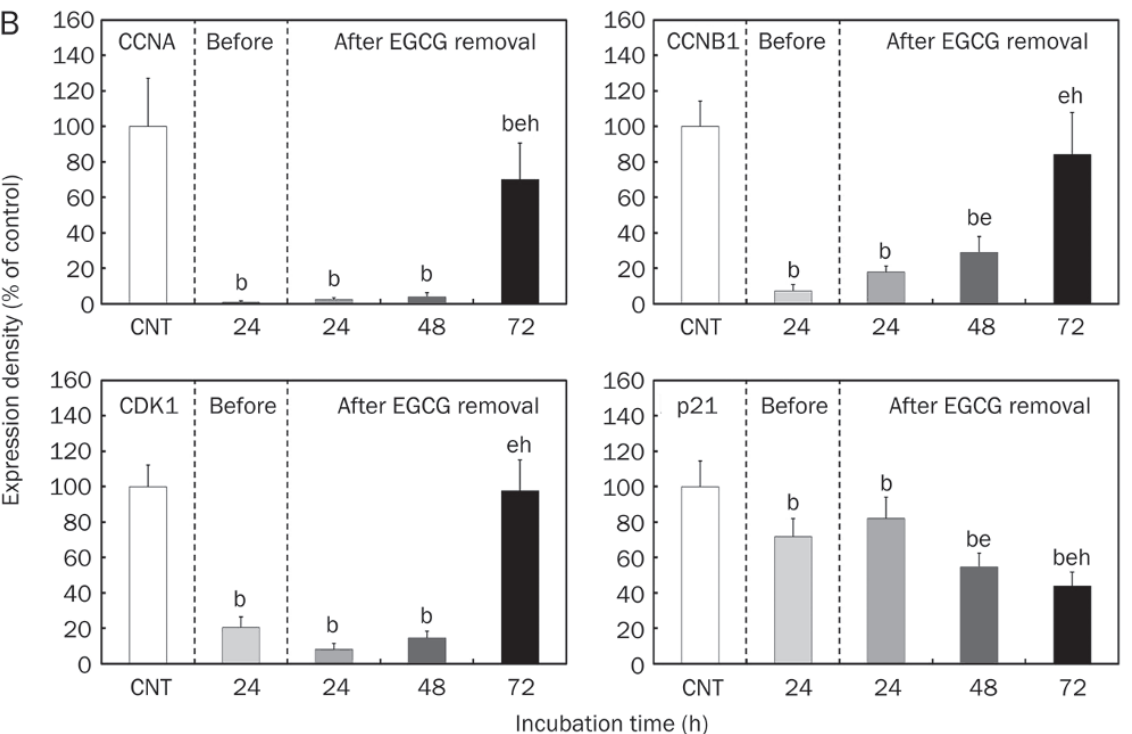

Figure 4. Effects of EGCG on expression of cell cycle-related proteins in nHDFs. After treatment with $200 \mu$ mol/L EGCG for 24 h, EGCG was removed from the cultured cells followed by further incubation for up to $72 \mathrm{~h}$. The expression of cell cycle-related proteins in $\mathrm{nHDFs}$ (A) was determined by Western blotting as described in Materials and methods. Results from representative experiments were normalized to GAPDH expression by densitometry. The quantitative results are shown in the right panel (B). The results are reported as a mean \pm SD and analyzed by a Tukey HSD test. ${ }^{\mathrm{b}} P<0.05$ vs non-treated control, ${ }^{\mathrm{e}} \mathrm{P}<0.05$ vs $24 \mathrm{~h}$ after EGCG treatment group, ${ }^{\mathrm{h}} \mathrm{P}<0.05$ vs 24 or $48 \mathrm{~h}$ after EGCG removal group.

within cells by virtue of competition with unlabelled EGCG for membrane binding sites. This cellular uptake pattern of FITC-EGCG in normal fibroblasts was completely different from the pattern in human fibrosarcoma (HT-1080) cells (as their cancer counterparts) ${ }^{[21]}$ and murine fibroblastic (L-929) cells (Supplementary Figure S1) ${ }^{[20]}$. Additional fluorescence microscopic observation demonstrated the time course of cellular uptake of FITC-EGCG in cultured nHDFs. FITC-EGCG was clearly observed in both the membrane and the cytoplasm of the cells until $10 \mathrm{~d}$, despite the removal of FITC-EGCG from the culture medium (Supplementary Figure S2).

Confocal micrographs of nHDFs detached due to apoptosis mediated by a higher dose $(400 \mu \mathrm{mol} / \mathrm{L})$ of FITC-EGCG showed that FITC-EGCG was still incorporated into the cytoplasm and even into the nucleus of the detached cells (Figure $5 \mathrm{~B})$. Both the membrane and the nucleus of detached nHDFs were too damaged to delineate due to apoptosis. Furthermore, the cells that were still attached but adversely affected by FITC-EGCG showed typical apoptotic appearances, including blebbing, nuclear swelling, and membrane deterioration, whereas they were subjected to the nuclear translocation of FITC-EGCG like their cancer counterparts ${ }^{[21]}$. These results imply that FITC-EGCG at an almost non-toxic concentration can be incorporated into the cytosol of the cells through binding to specific receptors and forming complexes with them. Furthermore, FITC-EGCG may be differentially translocated into the nucleus in nHDFs vs HT-1080 cells and L-929 cells, which contributes to the reversible regulation of the cell cycle and leads to cytoprotection.

\section{Discussion}

In the present study, we demonstrated that the dose-differential effects of EGCG were associated with the reversible regulation of the expression of cell cycle-related genes and proteins in nHDFs. Although several studies comparing the effects of EGCG on cancer cells and their normal counterparts have been reported previously ${ }^{[5-7]}$, it has not been clear whether these pharmacological effects of EGCG are related to the reversible regulation of the cell cycle mediated by EGCG incorporated into the cells. Natural dietary anti-cancer chemopreventive compounds, such as polyphenols (eg, EGCG and curcumin), isothiocyanates (eg, sulforaphane and phenethyl isothiocyanate) and their combinations, are known to possess redox-mediated differential signaling mechanisms that lead to cytoprotection of normal cells $v$ s cytotoxicity in tumor cells ${ }^{[22]}$. Our studies have shown that EGCG did not induce growth inhibition, cell cycle deregulation, or apoptosis of normal fibroblasts at $200 \mu \mathrm{mol} / \mathrm{L}$, which is a concentration that would be high enough to affect their cancer counterparts (Figures 1 and 2). This result suggests that EGCG or its derivatives can be developed as agents to selectively eliminate cancer cells without affecting normal cells. Other previous studies have primarily used concentrations of 40 to $100 \mu \mathrm{mol} / \mathrm{L}$ to demonstrate differential effects of EGCG on cancerous cells $v s$ their normal counterparts ${ }^{[5-7]}$. Even these concentrations are not physiological ${ }^{[23]}$, but provide a framework for understanding the differential effects of EGCG. Here, we employed 200 $\mu \mathrm{mol} / \mathrm{L}$ as a critical concentration to define the mechanistic basis of the differential effects of EGCG. Although this concentration is much higher than that used in other studies, 
A After $24 \mathrm{~h}$ of treatment with $100 \mu \mathrm{mol} / \mathrm{L}$ FITC-EGCG

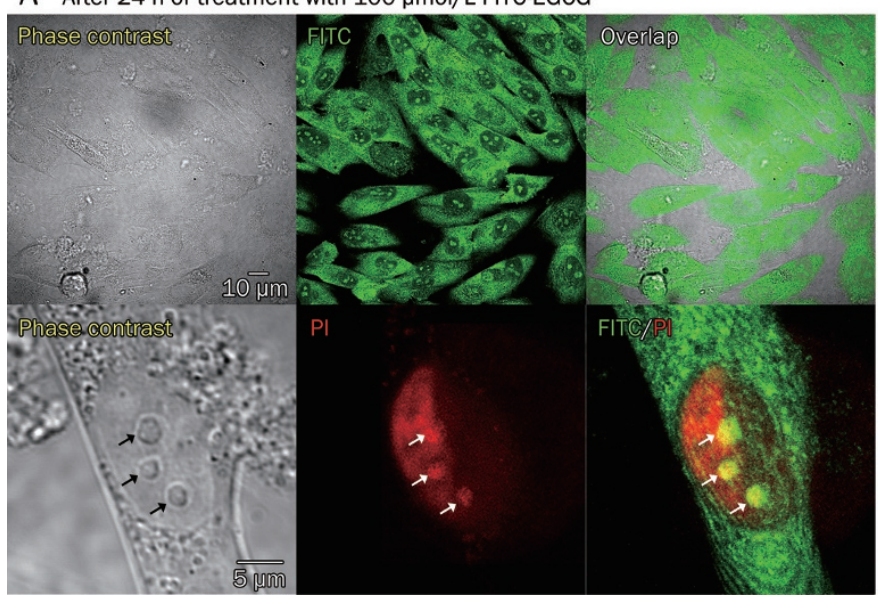

B After $24 \mathrm{~h}$ of treatment with $400 \mu \mathrm{mol} / \mathrm{L}$ FITC-EGCG

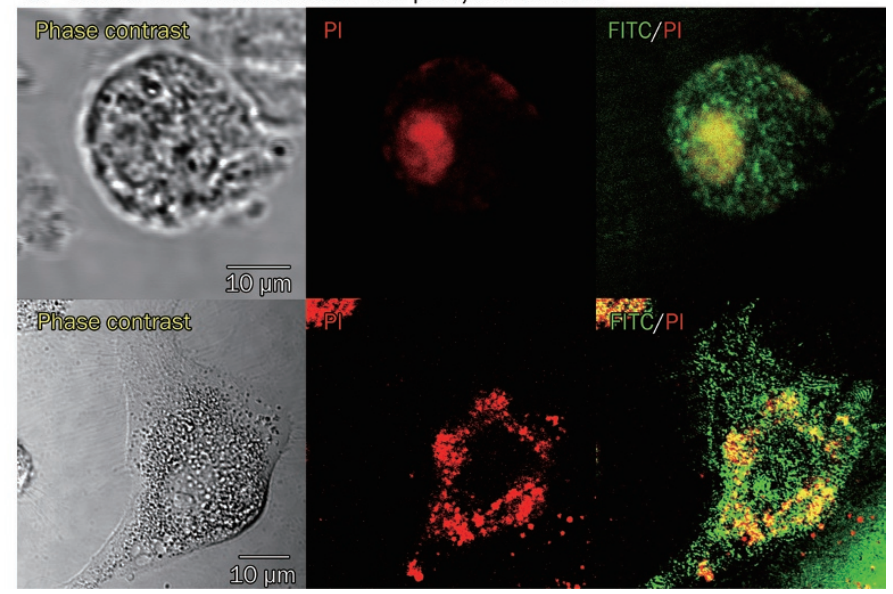

Figure 5. Cellular uptake of FITC-EGCG in nHDFs. Cells were incubated with $100 \mu \mathrm{mol} / \mathrm{L}$ (A) and $400 \mu \mathrm{mol} / \mathrm{L}$ (B) FITC-EGCG for $24 \mathrm{~h}$ and then observed under a confocal laser scanning microscope. The micrographs shown in this figure are representative of 6 independent experiments, showing similar results [Original magnification: $\times 500$ and $\times 2000$ (A, upper and lower, respectively), and $\times 1800$ (B)]. Arrows in A indicates FITC-EGCG translocated into the nucleus.

the $\mathrm{LC}_{50}$ value of EGCG on growth inhibition was estimated to be approximately $600 \mu \mathrm{mol} / \mathrm{L}$ in nHDFs (Supplementary Table S4). Moreover, HT-1080 cells were more sensitive to EGCG and had a much lower LC $_{50}$ value $(200 \mu \mathrm{mol} / \mathrm{L})$ than that of $\mathrm{nHDFs}^{[21]}$. Therefore, $200 \mu \mathrm{mol} / \mathrm{L}$ EGCG was considered suitable for understanding the dose-differential effects of EGCG on normal vs cancer fibroblasts. These results suggest the possibility that appropriate concentrations of EGCG may inhibit the growth of certain cancer cells, but have no apparent adverse effect on the growth of their normal counterparts.

We also showed that EGCG displayed a dose-differential

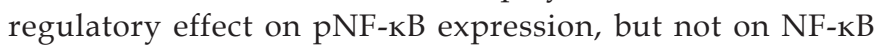
expression (Figure 3). Perkins et al have found that specific CDKs regulate NF-KB-mediated transcriptional activation through interactions with the coactivator p300 and that the transcriptional activation domain of RelA (p65) interacts with an amino-terminal region of p300 that is distinct from a carboxyl-terminal region of p300 required for binding to the CCNE-CDK2 complex ${ }^{[24]}$. According to their report, CDKN1A or a dominant negative CDK2, which inhibits p300-associated CCNE-CDK2 activity, stimulates NF-kB-dependent gene expression, and the interaction of NF-KB and CDKs through the p300 and CREB-binding protein coactivators provides a mechanism for the coordination of transcriptional activation with cell cycle progression. Increasing evidence has indicated that the development of selective NF-KB inhibitors may represent a promising therapeutic tool to sensitize tumor cells to apoptosis and increase the efficacy of conventional anticancer drugs in a wide spectrum of malignancies ${ }^{[25]}$.

In general, cDNA microarray analysis provides extensive information on global gene expression. Indeed, the data presented here demonstrated the global regulation of multiple genes involved in cell survival, cell cycle, and apoptosis that are considered some of the most important factors for cytoprotection $^{[16]}$. The genes that changed over 2-fold, namely either $\geq 2$ or $\leq 1 / 2$, constituted only $5.5 \%$ (84 genes) of the genes with statistical reliability (Table 1 and Supplementary Tables S1-S3). Our findings from the cDNA microarray showed that 20\% (10 genes) of cell cycle-related genes were strongly affected by EGCG treatment. Among the cell cycle-related genes, those that were the most affected by EGCG treatment were CCNs and CDKs directly involved in cell cycle regulation. Interestingly, a substantial difference in the response to EGCG among the cell cycle-related genes was present. CCNA2, CCNB1/ $B 2$, and CDK1, essential for the $\mathrm{G}_{2} / \mathrm{M}$ phase transition, were strongly suppressed, whereas CCNC, CCND1/3, CDK2, and $C D K 6$, essential for $G_{1}$ phase and the $G_{1} / S$ phase transition, were less suppressed (Tables 1 and 2). This cell cycle delay at the $G_{0} / G_{1}$ phase showed a proper correlation with the result of the cell cycle assay (Figure 1B). Based on the results of a cell growth assay and cytometry, cellular responses such as proliferation and cell cycle progression can be predicted to be regulated by cell cycle-related genes that were changed over 2-fold upon EGCG treatment. Therefore, the reversible effects of EGCG may allow its safe usage in cell protection for chemotherapy ${ }^{[16,18]}$. Our hypothesis was further verified by finding highly positive correlations with the results from immunoblotting that showed similar patterns to those of the microarray data (Figure 4). Removal of EGCG from the culture medium restored the expression of cell cycle-related proteins to the levels before EGCG treatment, which suggests that the effect of EGCG on the cell cycle is reversible. EGCG reduced the number of proliferating $\mathrm{nHDFs}$ cells by slowing the transit through the $S$ and $G_{2} / M$ phases, by delaying cell cycle at the $G_{1}$ phase, or even by inducing apoptotic cell death.

We also showed that the cellular uptake of FITC-EGCG in normal fibroblasts was different from that in a fibroblastic cell line (Figure 5 and Supplementary Figure S1) in their cancer counterparts (HT-1080 cells) ${ }^{[21]}$. These cellular uptake patterns were involved as important contributors to the dose- 
differential growth inhibitory, cell cycle regulatory, and apoptotic responses of EGCG. Although the exact mechanism of the incorporation of EGCG into cells has not been fully elucidated, some evidence has been reported that EGCG is bound to the membrane and incorporated into the cytosol and the nucleus of cancer cell lines (such as PC-9 and HT-29 cells) treated with $\left[{ }^{3} \mathrm{H}\right] \mathrm{EGCG}^{[8,26]}$. These quantitative results using $\left[{ }^{3} \mathrm{H}\right]$ EGCG agree well with our qualitative results showing that the FITC-EGCG was incorporated into the cytosol of fibroblastic cells ${ }^{[20,21]}$. However, the nuclear translocation pattern of FITC-EGCG was quite different in nHDFs vs HT-1080 cells and L-929 cells (Supplementary Figure S2), which would lead to the reversible regulation of the cell cycle and cytoprotection of normal fibroblasts. These phenomena can be related in part to the intrinsic characteristics of EGCG as a polyphenolic compound. EGCG is amphipathic and easily binds to extracellular matrices, lipid membranes or any type of intracellular protein $^{[27-29]}$. Therefore, EGCG seems to be able to be incorporated into the cytosol of fibroblastic cells and further translocated into the nucleus and may modulate the exogenous signals directed to genes required for the survival and apoptosis of the cells. Recent studies have demonstrated that expression of the metastasis-associated $67 \mathrm{kDa}$ laminin receptor might confer EGCG responsiveness to cancer cells at physiologically relevant concentrations, suggesting that the gallate moiety of EGCG may be critical for receptor binding and subsequent activity ${ }^{[30,31]}$. Moreover, the observation that nucleic acids extracted from catechin-treated cells were colored implied that because both galloyl and catechol groups of EGCG were essential for DNA and RNA binding, both groups seemed to hold strands of DNA and RNA via their branching structure ${ }^{[2,33]}$.

Taking these results into consideration, we conclude that EGCG internalized into normal fibroblasts can dose-differentially regulate the expression of cell cycle-related genes and proteins and exert cytoprotective effects on the cells through a feasible mechanism of action (Supplementary Figure S3). This dose-differential regulatory activity of EGCG may be exploited to craft strategies using EGCG for the cytoprotection of normal cells and the chemoprevention of cancer cells.

\section{Abbreviations}

CCN, Cyclin; CDC, cell division cycle; CDK, cyclin-dependent kinase; CDKN1A, CDK inhibitor 1A; CKS2, CDC28 protein kinase regulatory subunit 2; EGCG, (-)epigallocatechin-3gallate; FITC, fluorescein isothiocyanate; FITC-EGCG, FITCconjugated EGCG; GAPDH, glyceraldehyde-3-phosphate dehydrogenase; nHDFs, neonatal human dermal fibroblasts; PBS, phosphate-buffered saline; pNF-kB/p65, phosphorylated nuclear factor-kB/p65; PI, propidium iodide; PKMYT1, protein kinase, membrane associated tyrosine/threonine 1; SD, standard deviation; WST-8, water soluble tetrazolium salt.

\section{Acknowledgements}

This work was supported in part by a grant from the Korea Healthcare Technology R\&D Project, Ministry for Health, Welfare \& Family Affairs, Republic of Korea (A085136), a grant from the Fundamental R\&D Program for Core Technology of Materials funded by the Ministry of Knowledge Economy, Republic of Korea (K0006028), and the Korea Research Foundation Grant funded by the Korean Government (MOEHRD, Basic Research Promotion Fund) (KRF-2008-331-D00754).

\section{Author contribution}

Dong-Wook HAN, Mi Hee LEE, and Jong-Chul PARK conceived and designed the study; Dong-Wook HAN and Mi Hee LEE performed the research and analyzed the data; DongWook HAN, Hak Hee KIM, and Suong-Hyu HYON wrote the manuscript; Jong-Chul PARK edited the manuscript and provided the funding support for the studies.

\section{Supplementary information}

Additional experimental details for the microarray analysis and cytotoxicity assay are provided with the results for significantly down-regulated cell cycle-related genes in nHDFs after EGCG treatment (Table S1), significantly down-regulated/ up-regulated apoptosis-related genes in nHDFs after EGCG treatment (Table S2), significantly down-regulated/up-regulated signal transduction-related genes in NHDFs after EGCG treatment (Table S3), and $\mathrm{LC}_{50}$ values of EGCG against various phenotypes of primary cells or cell lines (Table S4). Other experimental details for confocal and fluorescence microscopy are provided with the results for the cellular uptake of FITCEGCG in L-929 cells (Figure S1) and the time course of cellular uptake of FITC-EGCG in cultured nHDFs (Figure S2) as well as a discussion of the feasible mechanism of action of EGCG (Figure S3).

These are available at Acta Pharmacologica Sinica website of NPG.

\section{References}

$1 \mathrm{Na} \mathrm{HK}$, Surh YJ. Intracellular signaling network as a prime chemopreventive target of (-)-epigallocatechin gallate. Mol Nutr Food Res 2006; 50: 152-9.

2 Khan N, Afaq F, Saleem M, Ahmad N, Mukhtar H. Targeting multiple signaling pathways by green tea polyphenol (-)-epigallocatechin-3gallate. Cancer Res 2006; 66: 2500-5.

3 Kwon KH, Barve A, Yu S, Huang MT, Kong AN. Cancer chemoprevention by phytochemicals: potential molecular targets, biomarkers and animal models. Acta Pharmacol Sin 2007; 28: 1409-21.

4 Nair S, Barve A, Khor TO, Shen GX, Lin W, Chan JY, et al. Regulation of Nrf2- and AP-1-mediated gene expression by epigallocatechin-3gallate and sulforaphane in prostate of Nrf2-knockout or C57BL/6J mice and PC-3 AP-1 human prostate cancer cells. Acta Pharmacol Sin 2010; 31: 1223-40.

5 Ahmad N, Feyes DK, Nieminen AL, Agarwal R, Mukhtar H. Green tea constituent epigallocatechin-3-gallate and induction of apoptosis and cell cycle arrest in human carcinoma cells. J Natl Cancer Inst 1997; 89: 1881-6.

6 Ahmad N, Gupta S, Mukhtar H. Green tea polyphenol epigallocatechin-3-gallate differentially modulates nuclear factor-kB in cancer cells versus normal cells. Arch Biochem Biophys 2000; 376: 338-46.

7 Chen ZP, Schell JB, Ho CT, Chen KY. Green tea epigallocatechin gallate shows a pronounced growth inhibitory effect on cancerous cells but not on their normal counterparts. Cancer Lett 1998; 129: 
173-9.

8 Okabe S, Suganuma M, Hayashi M, Sueoka E, Komori A, Fujiki H. Mechanisms of growth inhibition of human lung cancer cell line, PC-9, by tea polyphenols. Jpn J Cancer Res 1997; 88: 639-43.

9 Kohri T, Nanjo F, Suzuki M, Seto R, Matsumoto N, Yamakawa M, et al. Synthesis of $(-)-\left[4-{ }^{3} \mathrm{H}\right]$ epigallocatechin gallate and its metabolic fate in rats after intravenous administration. J Agric Food Chem 2001; 49: 1042-8.

10 Suganuma M, Okabe S, Oniyama M, Tada $\mathrm{Y}$, Ito H, Fujiki H. Wide distribution of $\left[{ }^{3} \mathrm{H}\right](-)$-epigallocatechin gallate, a cancer preventive tea polyphenol, in mouse tissue. Carcinogenesis 1998; 19: 1771-6.

11 Swezey RR, Aldridge DE, LeValley SE, Crowell JA, Hara Y, Green CE. Absorption, tissue distribution and elimination of $4-\left[{ }^{3} \mathrm{H}\right]$-epigallocatechin gallate in beagle dogs. Int J Toxicol 2003; 22: 187-93.

12 Ahmad N, Gupta S, Husain MM, Heiskanen KM, Mukhtar H. Differential antiproliferative and apoptotic response of sanguinarine for cancer cells versus normal cells. Clin Cancer Res 2000; 6: 1524-8.

13 Donnerstag B, Ohlenschlager G, Cinatl J, Amrani M, Hofmann D, Flindt $\mathrm{S}$, et al. Reduced glutathione and S-acetylglutathione as selective apoptosis-inducing agents in cancer therapy. Cancer Lett 1996; 110: 63-70.

14 Khan N, Adhami VM, Mukhtar H. Apoptosis by dietary agents for prevention and treatment of cancer. Biochem Pharmacol 2008; 76 : 1333-9.

15 Ramos S. Cancer chemoprevention and chemotherapy: dietary polyphenols and signalling pathways. Mol Nutr Food Res 2008; 52 : 507-26.

16 Bae JY, Kanamune J, Han DW, Matsumura K, Hyon SH. Reversible regulation of cell cycle-related genes by epigallocatechin gallate for hibernation of neonatal human tarsal fibroblasts. Cell Transplant 2009; 18: 459-69.

17 Hyon SH, Kim DH. Hibernation of mammalian cells at a living body temperature. Biotechnol Bioprocess Eng 2001; 6: 289-92.

18 Matsumura K, Kim JY, Tsutsumi S, Hyon SH. Hibernation, reversible cell growth inhibition by epigallocatechin-3-O-gallate. J Biotechnol 2007; 127: 758-64.

19 Park JC, Park BJ, Suh H, Park BY, Rah DK. Comparative study on motility of the cultured fetal and neonatal dermal fibroblasts in extracellular matrix. Yonsei Med J 2001; 42: 587-94.

20 Han D-W, Matsumura K, Kim B, Hyon SH. Time-dependent intracellular trafficking of FITC-conjugated epigallocatechin-3-O-gallate in L-929 cells. Bioorg Med Chem 2008; 16: 9652-9.

21 Lee MH, Han DW, Hyon SH, Park JC. Apoptosis of human fibrosarcoma
HT-1080 cells by epigallocatechin-3-O-gallate via induction of p53 and caspases as well as suppression of $\mathrm{Bcl}-2$ and phosphorylated nuclear factor-KB. Apoptosis 2011; 16: 75-85.

22 Nair S, Li W, Kong AN. Natural dietary anti-cancer chemopreventive compounds: redox-mediated differential signaling mechanisms in cytoprotection of normal cells versus cytotoxicity in tumor cells. Acta Pharmacol Sin 2007; 28: 459-72.

23 Chow HH, Cai Y, Hakim IA, Crowell JA, Shahi F, Brooks CA, et al. Pharmacokinetics and safety of green tea polyphenols after multipledose administration of epigallocatechin gallate and polyphenon $E$ in healthy individuals. Clin Cancer Res 2003; 9: 3312-9.

24 Perkins ND, Felzien LK, Betts JC, Leung K, Beach DH, Nabel GJ. Regulation of NF-KB by cyclin-dependent kinases associated with the p300 coactivator. Science 1997; 275: 523-7.

25 Piva R, Belardo G, Santoro MG. NF-kB: a stress-regulated switch for cell survival. Antioxid Redox Signal 2006; 8: 478-86.

26 Hong J, Lu H, Meng X, Ryu JH, Hara Y, Yang CS. Stability, cellular uptake, biotransformation, and efflux of tea polyphenol (-)-epigallocatechin-3-gallate in HT-29 human colon adenocarcinoma cells. Cancer Res 2002; 62: 7241-6.

27 Palermo CM, Westlake CA, Gasiewicz TA. Epigallocatechin gallate inhibits aryl hydrocarbon receptor gene transcription through an indirect mechanism involving binding to a $90 \mathrm{kDa}$ heat shock protein. Biochemistry 2005; 44: 5041-52.

28 Uekusa Y, Kamihira M, Nakayama T. Dynamic behavior of tea catechins interacting with lipid membranes as determined by NMR spectroscopy. J Agric Food Chem 2007; 55: 9986-92.

29 Yang D, Liu J, Tian C, Zeng Y, Zheng YH, Fang Q, et al. Epigallocatechin gallate inhibits angiotensin II-induced endothelial barrier dysfunction via inhibition of the p38 MAPK/HSP27 pathway. Acta Pharmacol Sin 2010; 31: 1401-6.

30 Tachibana H, Koga K, Fujimura Y, Yamada K. A receptor for green tea polyphenol EGCG. Nat Struct Mol Biol 2004; 11: 380-1.

31 Umeda D, Yano S, Yamada K, Tachibana H. Green tea polyphenol epigallocatechin-3-gallate signaling pathway through $67-\mathrm{kDa}$ laminin receptor. J Biol Chem 2008; 283: 3050-8.

32 Kuzuhara T, Sei Y, Yamaguchi K, Suganuma M, Fujiki H. DNA and RNA as new binding targets of green tea catechins. J Biol Chem 2008; 281: 17446-56.

33 Kuzuhara T, Tanabe A, Sei Y, Yamaguchi K, Suganuma M, Fujiki H. Synergistic effects of multiple treatments, and both DNA and RNA direct bindings on, green tea catechins. Mol Carcinog 2007; 46: 640-5. 